\title{
Interference effects in second-harmonic generation within an optical cavity
}

\author{
Ling-An Wu and H. J. Kimble \\ Department of Physics, University of Texas at Austin, Austin, Texas 78712
}

\begin{abstract}
Received August 29, 1984; accepted January 4, 1985
An experiment is described that investigates certain interference effects for second-harmonic generation within a resonant cavity. By employing a noncollinear geometry, the phases of two fundamental beams from a frequencystabilized dye laser can be controlled unrestricted by the boundary conditions imposed in an optical cavity containing a KDP crystal and resonant at the second harmonic. The fundamental beams are either traveling or standing waves and generate either one or two coherent sources of ultraviolet radiation within the cavity. The experiment demonstrates explicitly the dependence of second-harmonic phase on the fundamental phases and the dependence of coupling efficiency on the overlap of the harmonic polarization wave with the cavity-mode function. The measurements agree well with a simple theory.
\end{abstract}

\section{INTRODUCTION}

There has recently been a renewed interest in parametric interactions inside resonant cavities both because of the complex dynamical behavior that may be exhibited ${ }^{1,2}$ and because of the quantum-statistical properties of the radiation that may be produced by such cavities. ${ }^{3-5}$ In particular, continuouswave systems are considered in which single modes of fundamental and harmonic radiation interact within an interferometer that is nearly resonant at the two frequencies involved. It is, however, sometimes overlooked that the manner in which cavity modes will influence each other can depend strongly on the spatial overlap of the nonlinear polarization and the cavity-mode functions. Since the radiated harmonic wave lags the polarization wave by $90^{\circ}$, the mode functions must exhibit a similar phase relation or else the coupling between them will be small.

These ideas are of course well known and were established in the earliest experiments in nonlinear optics, especially in the context of second-harmonic generation and parametric oscillation in resonant cavities. ${ }^{6-14}$ In their early theoretical treatment of the coupling between the fundamental and second-harmonic modes of a confocal cavity, Kingston and $\mathrm{McWhorter}^{6}$ were aware of the difficulty of experimentally obtaining simultaneous resonance of both frequencies. Oshman and $\mathrm{Harris}^{8}$ predicted self-pulsing behavior in a doubly resonant parametric oscillator within a laser cavity, and this was later observed experimentally by Smith and Parker. ${ }^{9}$ Smith $^{10}$ also discussed theoretically the case of second-harmonic generation within a laser cavity. Gonzalez et al. ${ }^{11}$ conducted an experiment on intracavity harmonic generation that involved phase effects. A detailed theoretical treatment of phase effects in intracavity second-harmonic generation with some experimental results was given more recently by Volosov et al. ${ }^{12}$ Extensive reviews regarding the operation of optical parametric oscillators are available. ${ }^{13,14}$

In an attempt to demonstrate somewhat more explicitly the effects of relative phase on the coupling of fundamental and harmonic fields, we have designed an experiment in which the phases of two incident fundamental beams and the generated second-harmonic beam can be varied independently. The harmonic beam is allowed to resonate in a cavity noncollinear with the fundamental beams, and the overall length and absolute position of the cavity relative to the doubling crystal can be varied. In terms of the coupling coefficient $\kappa$ defined in Refs. 1,6 , and 8 , we are able to vary $\kappa$ by a simple change of phase of the fundamental, thus modulating the intensity of the second harmonic over a large range. Preliminary to a discussion of our experiment in Section 3, we recall in Section 2 well-known results for noncollinear frequency doubling and apply the formalism to the specific geometry that we wish to consider.

\section{NONCOLLINEAR SECOND-HARMONIC GENERATION}

In noncollinear second-harmonic generation two fundamental beams interact inside a birefringent crystal to produce the second harmonic along the bisector of the beams, as shown in Fig. 1. Note that the angle $2 \beta$ is the angle of intersection between the two fundamental beams inside the crystal. In all the cases to be considered we will use the plane-wave approximation, which can be justified if the length of intersection is well within the Rayleigh length, and we will restrict our attention to the regime of small conversion efficiencies so that the fundamental beams may be considered to be constant in amplitude. Furthermore, we will neglect beam walk-off, since in our experiment the crystal is very short and the resonance cavity for the second harmonic is separate from that for the fundamental.

The two fundamental fields $\left(\epsilon_{1}, \epsilon_{2}\right)$ in Fig. 1 are taken to have the form

$$
\begin{aligned}
\epsilon_{1}(\mathbf{r}, t)= & E_{1}(\mathbf{r}) \hat{e}_{1} \exp \left[-i\left(\omega t+K_{1} x \sin \beta\right.\right. \\
& \left.\left.-K_{1} z \cos \beta-\phi_{1}\right)\right]+ \text { c.c. }, \\
\epsilon_{2}(\mathbf{r}, t)= & E_{2}(\mathbf{r}) \hat{e}_{2} \exp \left[-i\left(\omega t-K_{2} x \sin \beta\right.\right. \\
& \left.\left.-K_{2} z \cos \beta-\phi_{2}\right)\right]+ \text { c.c. },
\end{aligned}
$$

where the complex amplitudes $\left(E_{1}, E_{2}\right)$ and wave vectors inside the crystal $\left(\mathbf{K}_{1}, \mathbf{K}_{2}\right)$ are chosen such that 


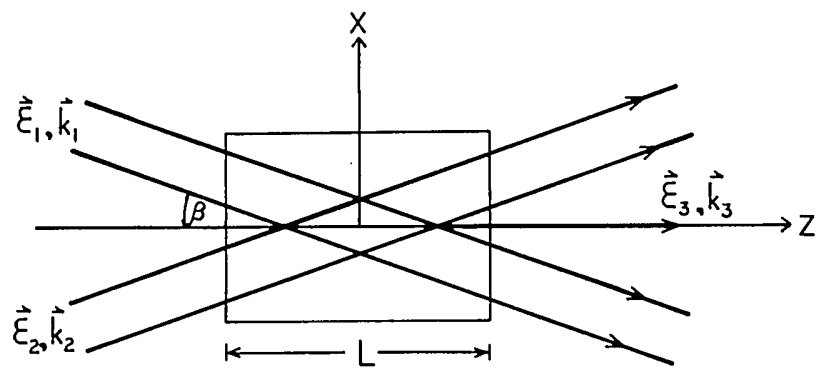

Fig. 1. Noncollinear second-harmonic generation of $\epsilon_{3}$ from two fundamental traveling waves $\left(\epsilon_{1}, \epsilon_{2}\right)$.

$$
\begin{gathered}
\left|E_{1}\right|=\left|E_{2}\right| \equiv E, \\
\left|\mathbf{K}_{1}\right|=\left|\mathbf{K}_{2}\right| \equiv K .
\end{gathered}
$$

$\hat{e}_{1}$ and $\hat{e}_{2}$ are unit polarization vectors, $\phi_{1}$ and $\phi_{2}$ are initial phases, and the $z$ and $x$ axes are along the bisectors of the incident rays as shown. For such an arrangement the induced polarization at the second harmonic is ${ }^{15-18}$

$$
\begin{aligned}
P_{i}^{(2 \omega)}(z, t)= & 2 \sum_{j k} \chi_{i j k} e_{1 j} e_{2 k} E_{1} E_{2} \\
& \times \exp \left[-i\left(2 \omega t-2 K z \cos \beta-\phi_{1}-\phi_{2}\right)\right]+\text { c.c., }
\end{aligned}
$$

with $\chi$ as the nonlinear susceptibility tensor as defined in Ref. 18. Assuming low conversion efficiency and negligible absorption losses in the crystal, we then have in cgs units for the complex amplitude $E^{(2 \omega)}$ of the second-harmonic field of wave vector $K_{3}$ propagating in the medium of index $n_{2 \omega}$,

$$
\begin{aligned}
\frac{\mathrm{d}}{\mathrm{d} z} E_{i}{ }^{(2 \omega)}(z)= & \frac{8 \pi i \omega}{n_{2 \omega} c} \sum_{j k} \exp \left[-i\left(K_{3}-2 K \cos \beta\right) z\right] \\
& \times \chi_{i j k} e_{1 j} e_{2 k} E^{2} \exp \left[i\left(\phi_{1}+\phi_{2}\right)\right]
\end{aligned}
$$

Integrating over the crystal length $L$, we obtain the wellknown result $16-20$

$$
E_{i}^{(2 \omega)}(L / 2)=i D_{i} E^{2} \exp \left[i\left(\phi_{1}+\phi_{2}\right)\right] \frac{\sin (\Delta K L / 2)}{(\Delta K L / 2)},
$$

or in the phase-matched case, $\Delta K \equiv K_{3}-2 K \cos \beta=0$,

$$
E_{i}^{(2 \omega)}(L / 2)=i D_{i} E^{2} \exp \left[i\left(\phi_{1}+\phi_{2}\right)\right]
$$

with

$$
D_{i} \equiv \frac{8 \pi \omega L}{n_{2 \omega} c} \sum_{j k} \chi_{i j k} e_{1 j} e_{2 k}
$$

Hence

$$
\left|E^{(2 \omega)}(L / 2)\right|^{2}=D^{2} E^{4}
$$

Given these basic results, we will now study the effects of changing the relative phases of the fundamental and second-harmonic beams for various traveling- and/or standingwave configurations.

\section{A. Two Second-Harmonic Beams from Two Fundamental Standing Waves}

In Fig. 2 the mirrors M1 and M2 reflect the fundamental beams back, thus setting up two standing waves, and an additional second harmonic wave is generated in the left-going direction by the reflected fundamental beams. Mirror MA then reflects this second-harmonic traveling wave $\mathbf{E}_{l}(2 \omega)$ $(-L / 2)$ back so that it interferes with the forward-generated second-harmonic beam. $h_{1}, h_{2}$ are the respective optical distances of M1, M2 from the center of the crystal. The amplitude of the right-generated second-harmonic wave on exiting the crystal face is the same as that given by Eq. (6),

$$
\mathbf{E}_{r}{ }^{(2 \omega)}(L / 2)=i \mathbf{D} E^{2} \exp \left[i\left(\phi_{1}+\phi_{2}\right)\right],
$$

where the suffix $r$ denotes right generated. The return fundamental waves have the form $E_{1} \exp [-i(\omega t-K x \sin \beta+K z$ $\left.\left.\cos \beta-\theta_{1}\right)\right]$ and $E_{2} \exp \left[-i\left(\omega t+K x \sin \beta+K z \cos \beta-\theta_{2}\right)\right]$, where $\theta_{1}$ and $\theta_{2}$ are the phases generated in transit to and from the mirrors M1, M2. By analogy with Eq. (6), the left-generated harmonic field leaving the left crystal face has amplitude

$$
\mathbf{E}_{l}^{(2 \omega)}(-L / 2)=i \mathbf{D} E^{2} \exp \left[i\left(\theta_{1}+\theta_{2}\right)\right]
$$

If we assume a phase change of $\phi_{r}$ on reflection at the mirrors M1 and M2 and take $k$ to be the wave vector in air, then

$$
\begin{aligned}
& \theta_{1}=\phi_{1}+2 k h_{1}+\phi_{r}, \\
& \theta_{2}=\phi_{2}+2 k h_{2}+\phi_{r} .
\end{aligned}
$$

Writing $h_{1}+h_{2}=H$ and substituting Eqs. (10) into Eq. (9), we have

$$
\mathbf{E}_{l}^{(2 \omega)}(-L / 2)=i \mathbf{D} E^{2} \exp \left[i\left(\phi_{1}+\phi_{2}+2 k H+2 \phi_{r}\right)\right] .
$$

After reflection from mirror MA with a phase change $\gamma$, the field arriving at the right crystal face is thus

$$
\begin{aligned}
\mathbf{E}_{l}^{(2 \omega)}(L / 2)= & i \mathbf{D} E^{2} \exp \left[i \left(\phi_{1}+\phi_{2}+2 k H\right.\right. \\
& \left.\left.+2 k_{3} s_{2}+2 \phi_{r}+\gamma\right)\right],
\end{aligned}
$$

with $K_{3}=n_{2 \omega} k_{3}$. Adding Eqs. (8) and (11), the total rightgoing field is

$$
\begin{aligned}
\mathbf{E}_{r+l}{ }^{(2 \omega)}(L / 2)= & i \mathbf{D} E^{2} \exp \left[i\left(\phi_{1}+\phi_{2}\right)\right] \\
& \times\left\{1+\exp \left[2 i\left(k H+k_{3} s_{2}+\Phi_{r} / 2\right]\right\},\right.
\end{aligned}
$$

with $\Phi_{r} \equiv 2 \phi_{r}+\gamma$, so that

$$
\left|\mathbf{E}_{r+l}{ }^{(2 \omega)}(L / 2)\right|^{2}=2(1+\cos \psi) D^{2} E^{4}
$$

where $\psi=2 k\left(h_{1}+h_{2}\right)+2 k_{3} s_{2}+\Phi_{r}$.

From Eq. (12) it can be seen that by varying the distance $h_{1}, h_{2}$, or $s_{2}$ the second-harmonic output may be modulated. Variation of $s_{2}$ of course gives rise to a trivial modulation of the second-harmonic field $E_{r+l}$ as in any interference experiment. The dependence of $E_{r+l}$ on $\left(h_{1}, h_{2}\right)$ is of more interest since it demonstrates the dependence of the second harmonic on the absolute phase of the fundamental. The maximum output possible is $4 D^{2} E^{4}$, which, as would be expected, is four

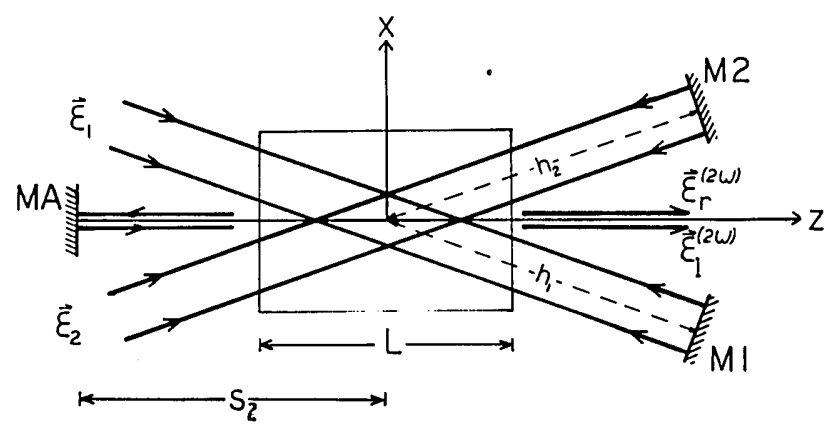

Fig. 2. Generation and superposition of two second-harmonic waves $\left[\epsilon_{r}^{(2 \omega)}, \epsilon_{l}^{(2 \omega)}\right]$ from two fundamental standing waves. 


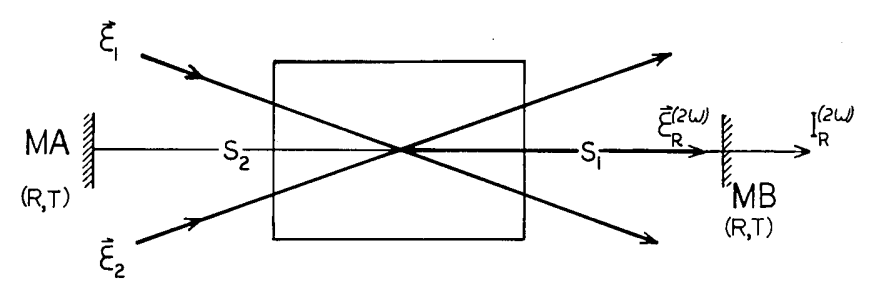

Fig. 3. Unidirectional generation of a single second-harmonic beam within a resonant cavity.

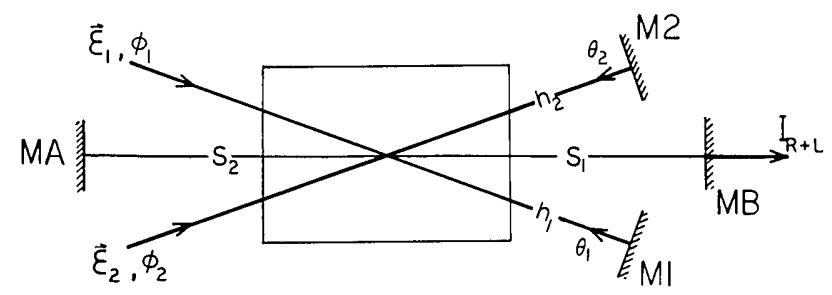

Fig. 4. Generation of two second-harmonic beams from two fundamental standing waves within a cavity resonant at the second harmonic.

times greater than for the single-beam case, but, on the other hand, complete destructive interference is also possible. It will be noted that only half the change in length of $s_{2}$ is required to produce the same phase change of $\psi$ as would be required by changing $h_{1}$ or $h_{2}$.

\section{B. Single-Harmonic Beam in a Resonant Cavity}

Consider next the case shown in Fig. 3 where the second harmonic is allowed to resonate in a cavity made up of two mirrors both of reflectivity $R$ and transmissivity $T$ at optical distances $s_{1}$ and $s_{2}$ from the center of the crystal. Let $\alpha / 2$ be the absorption loss per unit length of the crystal, which until now we have assumed to be negligible. At the crystal face $(z=$ $L / 2$ ) the right-going harmonic field in the cavity is given by

$$
\begin{aligned}
\mathbf{E}_{R}{ }^{(2 \omega)}(L / 2)= & \mathbf{E}_{r}{ }^{(2 \omega)}(L / 2)\left(1+r^{2} \exp \left[-\alpha L+2 i k_{3}\left(s_{1}+s_{2}\right)\right]\right. \\
& \left.+\left\{r^{2} \exp \left[-\alpha L+2 i k_{3}\left(s_{1}+s_{2}\right)\right]\right\}^{2}+\ldots\right) \\
& =\mathbf{E}_{r}{ }^{(2 \omega)}(L / 2) /[1-R \exp (-\alpha L+i \delta)],
\end{aligned}
$$

where $\mathbf{E}_{r}{ }^{(2 \omega)}(L / 2)$ is the same as that in Eq. (8), $r$ is the reflection coefficient for the mirror $\left(r=|r| e^{i \gamma}\right.$ with $\left.|r|^{2}=R\right)$, and $\delta=2 k_{3}\left(s_{1}+s_{2}\right)+2 \gamma$. The intensity transmitted through mirror MB is thus

$$
\begin{aligned}
I_{R}^{(2 \omega)} & \propto T\left|E_{R}(2 \omega)\right|^{2} \\
& \propto T D^{2} E^{4} /\left[\left(1-R e^{-\alpha L}\right)^{2}+4 R e^{-\alpha L} \sin ^{2}\left(k_{3} s+\gamma\right)\right],
\end{aligned}
$$

where $s=s_{1}+s_{2} . \quad I_{R}{ }^{(2 \omega)}$ thus displays the resonant structure associated with the Fabry-Perot interferometer.

\section{Two Harmonic Beams from Two Standing Waves in a Resonant Cavity}

With the configuration shown in Fig. 4 the harmonic output transmitted through mirror MB is given by the combination of expression (14) and Eq. (12), i.e.,

$$
I_{R+L}(2 \omega) \propto \frac{2(1+\cos \psi) T D^{2} E^{4}}{\left(1-R e^{-\alpha L}\right)^{2}+4 R e^{-\alpha L} \sin ^{2} \delta / 2} .
$$

Here we see that there are two phase factors involved, $\cos \psi$ and $\sin ^{2} \delta / 2$. Variation of $\delta$ corresponds to the usual scanning of a Fabry-Perot cavity as described in Subsection 2.B.
Variation of $\psi$ corresponds to variation of the coupling constant $\kappa$ since $\kappa^{2} \propto(1+\cos \psi)$. By suitable choice of $\psi$, the resonance peaks of $I_{R+L}{ }^{(2 \omega)}$ may be enhanced by a factor of 4 or extinguished altogether. The phase $\psi$ that produces this overall modulation of the Fabry-Perot peaks depends both on the phases of the fundamental beams and on the absolute position of the resonator relative to the fundamental standing-wave pattern inside the nonlinear crystal. This latter dependence may be seen by examining expression (15). For $\gamma=0$, resonance in the cavity requires that $s=s_{1}+s_{2}=$ $m\left(\lambda_{3} / 2\right)$ in the denominator, where $m$ is an integer. If this condition is satisfied and if the cavity is moved as a whole (keeping $s$ constant) along the $\pm z$ direction, then $\psi$ in the numerator will be changed through its dependence on $s_{2}$, again modulating the output intensity.

The results of the above calculation may be readily extended to the collinear case as well. For the simplest collinear geometry that might be imagined, the frequency-doubling crystal would be placed inside a standing-wave cavity formed by two mirrors that are highly reflecting at both $\omega$ and $2 \omega$. This cavity would then be driven by a single beam through one of the mirrors. Our analysis has shown that for this configuration almost no second harmonic would be generated. This is because the boundary condition demanding that the harmonic wave have nodes at the mirrors [as in Fig. 5(a)] is not commensurate with the fact that the radiated harmonic wave lags the polarization wave by $\pi / 2$ [as in Fig. 5(b)]. To satisfy the cavity boundary conditions one would have to introduce suitable dispersive elements between the ends of the crystal and the mirrors, ${ }^{10}$ thus shifting the phase of the harmonic by $(2 p+1)(\pi / 2)$ while maintaining a change in the fundamental of $q \pi$ (with $p, q$ both integers). As the relative position of the nodal patterns is changed, the coupling constant $\kappa$ of fundamental to second harmonic oscillates between a maximum and a minimum value since $\kappa$ is proportional to $\cos \psi / 2$. For Gaussian beams in a crystal of length comparable with the confocal parameter, similar considerations apply. ${ }^{7}$

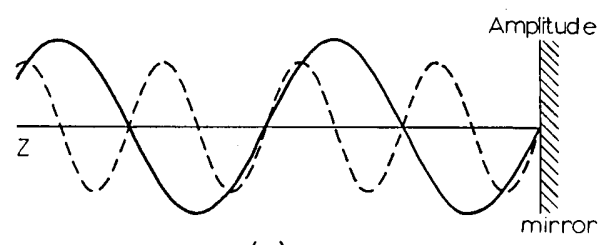

(a)

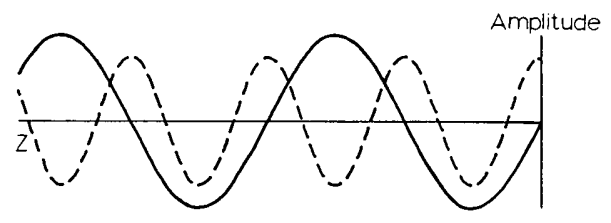

(b)

Fig. 5. Collinear frequency doubling in a Fabry-Perot cavity in which both the fundamental (solid line) and the second harmonic (dashed line) are resonant. (a) Reflection at cavity mirror showing relative phase of fundamental and harmonic standing waves. (b) Phase of generated second-harmonic field relative to the fundamental standing wave within the nonlinear crystal. Dispersive elements at the mirror itself or within the cavity must be used to compensate (a) relative to (b), otherwise little second harmonic will be coupled into the cavity mode. 


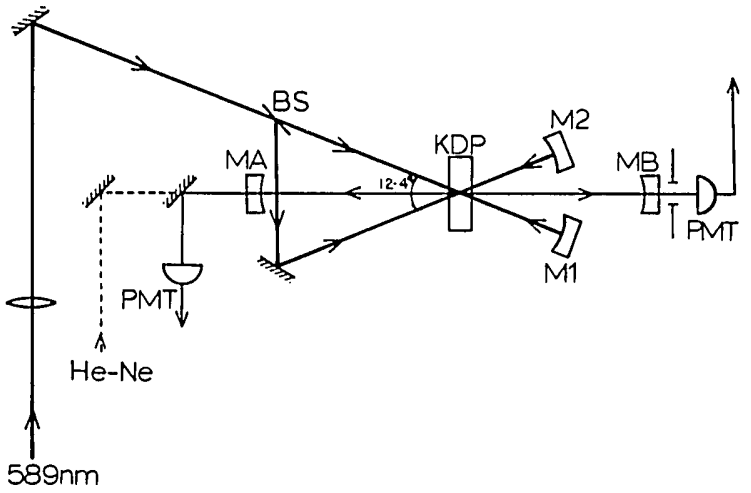

Fig. 6. Schematic of optical arrangement. Mirrors M1 and M2 are used to create two fundamental standing waves within the KDP crystal. Mirrors MA and MB form an optical resonator for the generated second harmonic at $295 \mathrm{~nm}$.

\section{EXPERIMENT}

We describe in this section a series of experiments conducted to investigate the interference in second-harmonic generation as discussed in the previous section. The optical arrangement employed is shown in Fig. 6. The source for the fundamental radiation is a single-mode dye laser that is frequency stabilized to $300 \mathrm{kHz}$ rms and polarized in the vertical plane. The fundamental beam is focused such that the two beams emerging from the beam splitter BS interact in the nonlinear crystal close to their Gaussian waists and such that the generated second harmonic is approximately mode matched to the cavity formed by mirrors MA and MB. Two totally reflecting mirrors M1 and M2 of radius of curvature $25 \mathrm{~cm}$ are used to retroreflect the fundamental beams. Distortion of the fundamental beam produced by an optical isolator causes the focused beams at the nonlinear crystal to have unequal waists in the horizontal and vertical directions. In the horizontal plane the apparent waists are $w_{h}{ }^{(\omega)}=129 \mu \mathrm{m}$ and $w_{h}{ }^{(2 \omega)}=95 \mu \mathrm{m}$ located 63 and $9 \mathrm{~mm}$ from the nonlinear crystal on the side of MA, while in the vertical plane $w_{v}{ }^{(\omega)}=185 \mu \mathrm{m}$ and $w_{v}{ }^{(2 \omega)}=139 \mu \mathrm{m}$ located 44 and $4 \mathrm{~mm}$ from the crystal on the side of MB. The crystal is well within the Rayleigh lengths of all waists. The second-harmonic cavity is $38 \mathrm{~cm}$ long and consists of mirrors $\mathrm{MA}$ and $\mathrm{MB}$ of radius of curvature $30 \mathrm{~cm}$ and reflectivity of 0.9988 at $295 \mathrm{~nm}$. The mirrors M1, M2, MA, MB are all mounted on cylindrical piezoelectric transducers.

The nonlinear medium is an antireflection-coated KDP crystal of dimensions $10 \mathrm{~mm} \times 10 \mathrm{~mm} \times 1.5 \mathrm{~mm}$ from Quantum Technology. The KDP crystal is cut for Type I angle phase matching with two noncollinear ordinary beams giving an extraordinary second-harmonic beam. The exterior angle between the fundamental beams is $12.4^{\circ}$, and maximum UV output is obtained when the crystal is rotated $3^{\circ}$ from its symmetric position. The calculated phase-matching angle of $68.7^{\circ}$ corresponds to a walk-off angle of about $1.2^{\circ}$. The total transmission losses through the crystal were measured with a Cary 17DX spectrophotometer to be $8.8 \%$ at $295 \mathrm{~nm}$.

The UV output power is maximized by adjusting the orientation and translational position of the crystal with respect to the fundamental beams, as well as by optimizing the overlap of the latter, in the absence of M1, M2, MA, and MB. The UV power is then measured as a function of the square of the incident power in one of the fundamental beam arms. Excellent linearity is obtained as expected from the lower power and hence low conversion efficiency $\left(\sim 1.3 \times 10^{-9} \mathrm{~W}\right.$ of UV from $50-\mathrm{mW}$ power in each arm) of our experiment. A theoretical calculation based on Refs. 20 and 21 with $\chi_{14}=2 d_{14}=2.2 \times$ $10^{-9} \mathrm{~cm} / \mathrm{dyn}^{1 / 2}$ or $\chi_{\text {ef }}=2.05 \times 10^{-9} \mathrm{~cm} / \mathrm{dyn}^{1 / 2}, L=1.5 \mathrm{~mm}$, $w=$ (average of horizontal and vertical radii at the center of the crystal) $=170 \mu \mathrm{m}$ gives the UV power to be $\sim 1.6 \times 10^{-9}$ W. The discrepancy between calculated and measured values is within the limits of experimental error. It should be pointed out that the interaction length of the beams, in our case $3 \mathrm{~mm}$, is actually longer than the crystal.

Given this description of the basic characteristics of the arrangement shown in Fig. 6, we now turn to a discussion of three experiments that illustrate the results derived in Section 2.
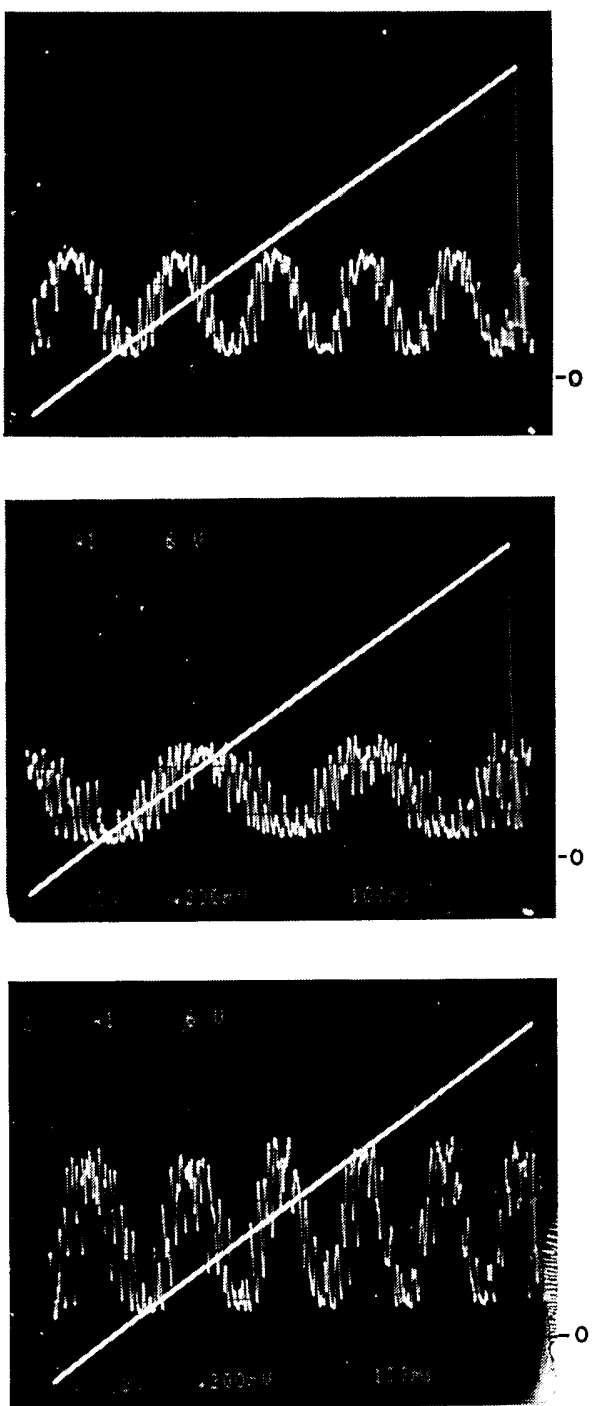

Fig. 7. Variation of intensity of second harmonic for the configuration illustrated in Fig. 2 and Eq. (12). In (a)-(c) the horizontal axis corresponds to voltage applied to a piezoelectric transducer (the diagonal line in the figure is the voltage versus time). The ordinate in each photograph is the UV intensity, with different sensitivities in (a)-(c). (a) Mirror MA is scanned, thus varying $\psi$ in Eq. (12) through the dependence on $s_{2}$ (ordinary two-beam interference). (b) Mirror M1 is scanned varying $\psi$ in Eq. (12) through the dependence on $h_{1}$ (phase of one fundamental beam). (c) Mirrors M1 and M2 are scanned simultaneously varying $\psi$ in Eq. (12) through the dependence on $\left(h_{1}+h_{2}\right)$ (phases of both fundamental beams). 


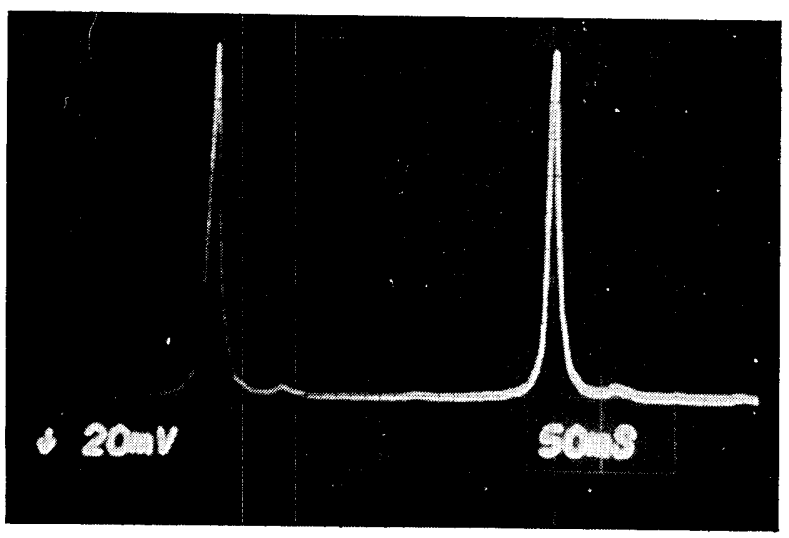

Fig. 8. Fabry-Perot resonances produced by a variation of $s_{2}$ and hence of the cavity length for the situation illustrated in Fig. 3 and expression (14).

\section{A. Two Second-Harmonic Beams from Two Fundamental Standing Waves}

Mirrors M1, M2, and MA are arranged as in Fig. 2, and each can be driven separately with a voltage ramp so that $h_{1}, h_{2}$, and $s_{2}$ in the phase $\psi$ of Eq. (12) can be separately scanned. When M1 and M2 are driven simultaneously from the same ramp, $\left(h_{1}+h_{2}\right)$ is scanned. Traces of the UV signal obtained versus transducer voltage, i.e., versus distance scanned, after the nonlinear responses of the various transducers are taken into account are shown in Figs. 7(a)-7(c). It can be seen that strong interference occurs between the two UV beams, with an oscillation period corresponding to a change of $\lambda_{3} / 2$ in $s_{2}$ or in $\left(h_{1}+h_{2}\right)$, but for $h_{1}$ alone being scanned, the oscillation period doubles, i.e., a change of $\lambda_{3}$ is required. This is in complete accordance with Eq. (12) and demonstrates the variation of phase of the second harmonic with phase of the fundamental. However, the minimum signal level never reaches zero. This is due to the fact that the backward-generated UV field $E_{l}{ }^{(2 \omega)}$ is greater than the forward field $E_{r}(2 \omega)$ $(L / 2)$, which is caused by mismatch at the mirrors M1 and M2 of the distorted Gaussian beam radii of the fundamental beams, resulting in tighter focusing of the latter on reflection. The erratic fine oscillations seen in the photographs are due to fluctuations in phase of the fundamental, caused by mechanical vibrations of mirrors. A similar experiment was reported by Yarborough et al., who used the dispersion of air to compensate for the phase shift between fundamental and second harmonic in a two-pass experiment. ${ }^{22}$

\section{B. Single Second-Harmonic Beam in a Resonant Cavity}

With the configuration of Fig. 3, we can scan mirror MA to produce UV resonance peaks of the Fabry-Perot cavity, as discussed in Subsection 2.B and illustrated in Fig. 8. Here the scanning of MA corresponds to a variation of $s_{2}$, with the time between two peaks corresponding to a change in distance of $0.5 \lambda_{3}$. The small peaks in the photograph correspond to transverse modes of the cavity that are excited by the nonideal waist configuration, as previously described. The measured finesse of 27 is somewhat lower than the calculated finesse of 35 , which is based solely on the losses at the crystal.

\section{Two Harmonic Beams from Two Standing Waves in a Resonant Cavity}

With the final arrangement of Fig. 4 and with MA again scanned, the height of the resonant UV peaks transmitted through MB can be varied, unlike the single-beam case above, by applying a de voltage to the transducers of M1, M2, or MB. The maximum height obtainable is 3.25 times greater than in the previous case, as may be seen by comparing Figs. 9(a) and 9(c). Such an increase is expected from the extra factor of 2(1 $+\cos \psi$ ) in expression (15) compared with expression (14). The reduced magnification factor of 3.25 instead of 4 may be attributed to the difference in amplitudes of the left- and right-generated UV fields. By suitably adjusting the dc voltages applied to M1 and/or M2 a minimum for the UV output can be obtained, as shown in Fig. 9(b). This minmum value is approximately 70 times smaller than the maximum signal shown in Fig. 9(a). We stress that the extinction of the second harmonic shown in Fig. 9(b) is due only to a change in phase of the fundamental through the dependence on $\left(\theta_{1}, \theta_{2}\right)$ and occurs even though the resonator length is tuned for
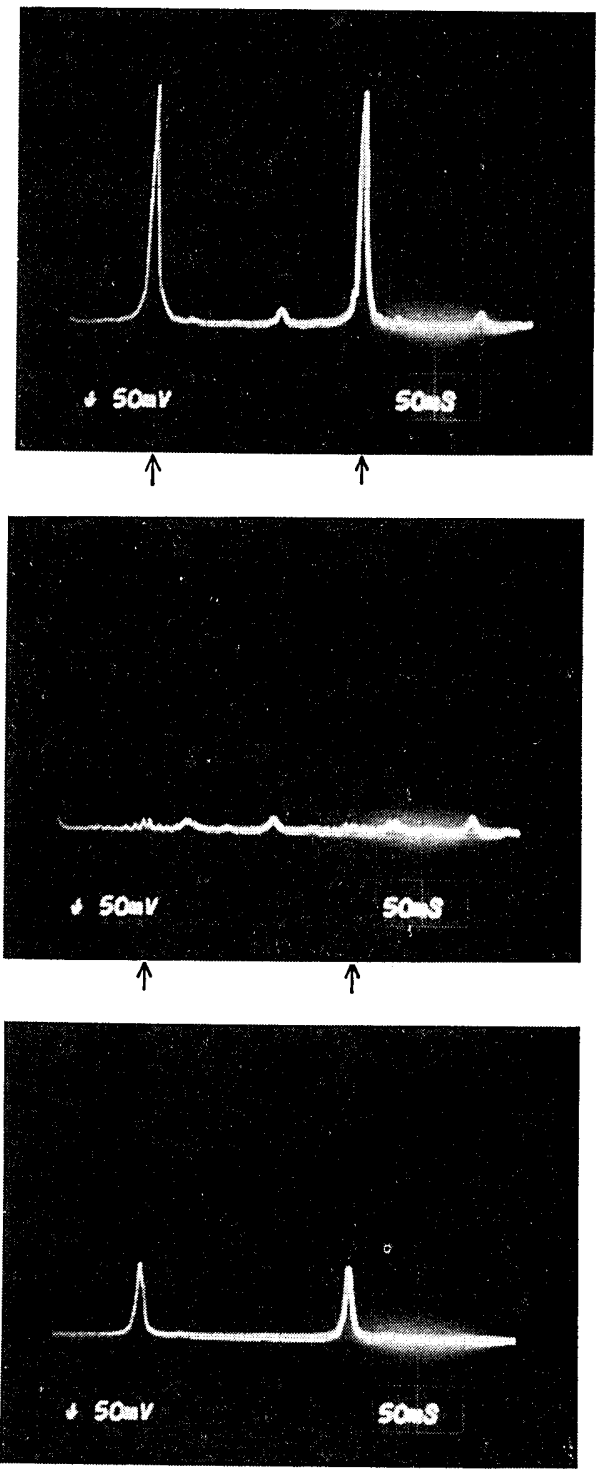

Fig. 9. Fabry-Perot resonances for the arrangement of Fig. 4 and expression (15). Second-harmonic power transmitted through mirror MB is displayed as a function of voltage applied to transducer of MA. (a) $\left(h_{1}, h_{2}\right)$ tuned for maximum UV signal. (b) $\left(h_{1}, h_{2}\right)$ tuned for minimum UV signal. (c) Resonance signal from a single UV wave, as in Fig. 8. The only distinction between (a) and (b) is the position of the mirrors M1, M2. 


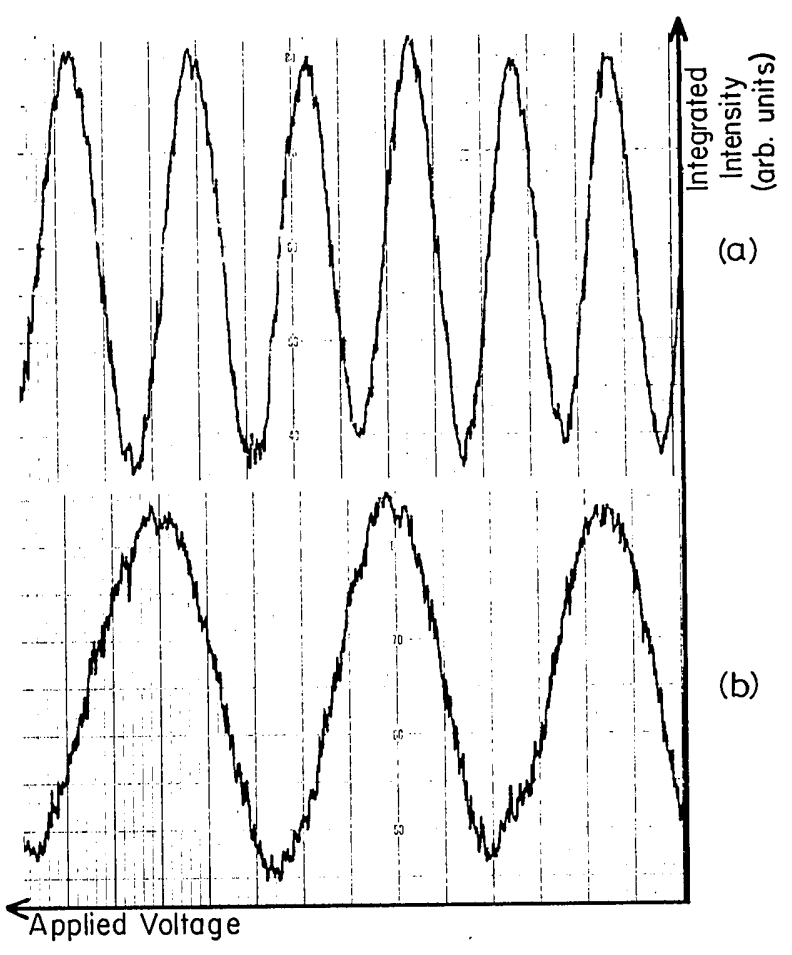

Fig. 10. Integrated second-harmonic power versus voltage on transducers of M1, M2 as discussed in the text. (a) $\left(h_{1}, h_{2}\right)$ are scanned simultaneously, and (b) $h_{1}$ is scanned alone to produce a modulation of coupling efficiency. The horizontal scale is such that 1 major division $\approx 58 \mathrm{~nm}$. When the nonlinear responses of the transducers are included, the peak-to-peak spacing is $\lambda_{3} / 2$ in (a) and $\lambda_{3}$ in (b).

resonance of the second harmonic [from expression (15), $\delta=$ $2 \pi m$ at the position of the arrows in Fig. 9].

To illustrate more quantitatively these dependences on relative phase we have recorded in Fig. 10 the integrated UV response versus drive voltage to the mirrors M1 and M2. The abscissa can be regarded as distance scanned by the mirrors, apart from the nonlinearities of the piezoelectrics. Mirrors M1 and M2 are driven at a rate of about $0.025 \lambda / \mathrm{sec}$, whereas MA is driven 1,000 times faster. This procedure corresponds to the simultaneous scanning of $\psi$ (slow) and $k_{3} s_{2}$ (fast) in expression (15), which may be written in full as

$$
I_{R+L}{ }^{(2 \omega)} \propto \frac{2\left\{1+\cos \left[2 k\left(h_{1}+h_{2}\right)+2 k_{3} s_{2}+\Phi_{r}\right]\right\} T D^{2} E^{4}}{\left(1-R e^{-\alpha L}\right)^{2}+4 R e^{-\alpha L} \sin ^{2}\left[k_{3}\left(s_{1}+s_{2}\right)+\gamma\right]} .
$$

The vertical signal in Fig. 10 represents the integrated UV output from the cavity over many sweeps of $s_{2}$. The peaks of the traces in Fig. 10 correspond to the time during which the heights of the Fabry-Perot peaks are at a maximum, i.e., whenever $\psi=2 k\left(h_{1}+h_{2}\right)+2 k_{3} s_{2}+\Phi_{r}=2 p \pi$. We see from expression (16) for $\gamma=\Phi_{r}=0$ that, every time $s=m\left(\lambda_{3} / 2\right)$, we have $s_{2}=m\left(\lambda_{3} / 2\right)-s_{1}$, so that $\psi$ may be written as $2[m \pi$ $\left.-k_{3} s_{1}+k\left(h_{1}+h_{2}\right)\right]$ whenever the harmonic cavity comes into resonance. In Fig. 10(a) $h_{1}$ and $h_{2}$ are moved together with $s_{1}$ fixed, producing a variation in the phases of the return fundamental beams and thus a modulation in the intensity of the second-harmonic resonance through the dependence on $\psi$ [expression (16)]. If only $h_{1}$ is scanned as in Fig. 10(b), the rate of change of phase is half as great. A similar trace to
Fig. 10(a) is obtained if $\psi$ is varied through its dependence on $s_{1}$. After correcting for the nonlinear response of the piezoelectric transducers, we find that the periods of the traces in Figs. 10(a) and 10(b) are the same as those in Fig. 7, again verifying the interference due to the phase $\psi$ in Eq. (12) and expression (15). We note in passing that the ratio of circulating UV power inside the cavity in Fig. 10(a) to that obtained in the absence of the mirrors MA, MB is 100 to 1 .

\section{CONCLUSION}

Our experiment has demonstrated that, in noncollinear second-harmonic generation with the nonlinear crystal inside a Fabry-Perot cavity, the second-harmonic output depends on the relative phases of the generated second-harmonic beams (as determined by the two fundamental beams) and the mode function of the resonant cavity. This statement translates into a sensitive dependence of the coupling efficiency $\kappa$ on the absolute position of the cavity relative to the fundamental beams and relative to the nonlinear crystal. One consequence of this dependence is that the simple procedure of detuning a resonator by changing its length will in certain geometries change the coupling coefficient as well. Although, as emphasized in Section 1, these ideas have been previously documented, we believe that the experiment just described represents a particularly clean and unambiguous demonstration of the phase relationships in nonlinear optics.

\section{ACKNOWLEDGMENT}

This research was supported by The Venture Research Unit of British Petroleum International.

\section{REFERENCES}

1. P.D. Drummond, K. J. McNeil, and D. F. Walls, "Nonequilibrium transitions in sub/second harmonic generation I. Semiclassical theory," Opt. Acta 27, 321 (1980); C. M. Savage and D. F. Walls, "Optical chaos in sub/second harmonic generation," Opt. Acta 30, 557 (1983).

2. P. Mandel and T. Erneux, "Amplitude self-modulation of intracavity second harmonic generation," Opt. Acta 29, 7 (1982).

3. P.D. Drummond, K. J. McNeil, and D. F. Walls, "Nonequilibrium transitions in sub/second harmonic generation II. Quantum theory," Opt. Acta 28, 211 (1981); G. Milburn and D. F. Walls, "Production of squeezed states in a degenerate parametric amplifier," Opt. Commun. 39, 401 (1981).

4. B. Yurke, "Use of cavities in squeezed-state generation," Phys. Rev. A 29, 408 (1984).

5. L. A. Lugiato, G. Strini, and F. de Martini, "Squeezed states in second harmonic generation," Opt. Lett. 8, 256 (1982).

6. R. H. Kingston and A. L. McWhorter, "Electromagnetic mode mixing in nonlinear media," Proc. IEEE 53, 4 (1965).

7. A. Ashkin, G. D. Boyd, and J. M. Dziedzic, "Resonant optical second harmonic generation and mixing,". IEEE J. Quantum Electron. QE-2, 109 (1966); G. D. Boyd and D. A. Kleinman, "Parametric interaction of focused Gaussian light beams," J. Appl. Phys. 39, 3597 (1968).

8. M. K. Oshman and S. E. Harris, "Theory of optical parametric oscillation internal to the laser cavity,"IEEE J. Quantum Electron. QE-4, 491 (1968).

9. R. G. Smith and J. V. Parker, "Experimental observation of and comments on optical parametric oscillation internal to the laser cavity," J. Appl. Phys. 41, 3401 (1970).

10. R. G. Smith, "Theory of intracavity optical second-harmonic generation," IEEE J. Quantum Electron. QE-6, 215 (1970).

11. D. G. Gonzalez, S. T. K. Nieh, and W. H. Steier, "Two-pass-in- 
ternal second-harmonic generation using a prism coupler," IEEE J. Quantum Electron. QE-9, 23 (1973).

12. V. D. Volosov, N. E. Kornienko, V. N. Krylov, A. I. Ryzhkov, and V. L. Strizhevskii, "Phase effects in intracavity optical second harmonic generation 1 . The case of free harmonic output from the cavity," Opt. Spectrosc. (USSR) 46, 64 (1979); V. D. Volosov, S. G. Karpenko, N. E. Kornienko, V. N. Krylov, A. A. Man'ko, and V. L. Strizhevskii, "Second-harmonic generation in a resonator," Sov. J. Quantum Electron. 5, 500 (1975).

13. R. G. Smith, "Optical parametric oscillators," in Laser Handbook, F. T. Arecchi and E. O. Schultz-Dubois, eds. (North-Holland, Amsterdam, 1972), p. 837.

14. R. L. Byer, "Optical parametric oscillators," in Quantum Electronics: A Treatise, H. Rabin and C. L. Tang, eds. (Academic, New York, 1975), Vol. 1, Part B, p. 587.

15. N. Bloembergen, Nonlinear Optics (Benjamin, New York, 1965).
16. Y. R. Shen, The Principles of Nonlinear Optics (Wiley, New York, 1984).

17. G. D. Boyd and D. A. Kleinman, "Parametric interaction of focused Gaussian light beams," J. Appl. Phys. 39, 3597 (1968).

18. F. Zernike and J. E. Midwinter, Applied Nonlinear Optics (Wiley, New York, 1973).

19. For boundless plane waves, the integration length would be the crystal length. In actual practice for finite beam widths, $L$ would be the length of the interaction region, and correction for the finite width in the $x$ direction would have to be made.

20. H. Fery and F. Hermann, "Noncollinear second harmonic generation in KDP," Opt. Commun. 8, 291 (1973).

21. F. Zernike, "Refractive Indices of ADP and KDP between 2000 $\AA$ and $1.5 \mu \mathrm{m}$, , J. Opt. Soc. Am. 54, 1215 (1964).

22. J. M. Yarborough, J. Falk, and C. B. Hitz, "Enhancement of optical second harmonic generation by utilizing the dispersion of air," Appl. Phys. Lett. 18, 70 (1971). 